\title{
An ischemia detection method based on artificial neural networks
}

\author{
Costas Papaloukas ${ }^{\mathrm{a}}$, Dimitrios I. Fotiadis, ${ }^{\mathrm{b}}{ }^{*}$, Aristidis Likas ${ }^{\mathrm{b}}$, \\ Lampros K. Michalis ${ }^{\mathrm{c}}$ \\ ${ }^{a}$ Department of Medical Physics, Medical School, University of Ioannina, Ioannina, Greece \\ ${ }^{\mathrm{b}}$ Department of Computer Science, University of Ioannina, Ioannina, Greece \\ ${ }^{\mathrm{c}}$ Department of Cardiology, Medical School, University of Ioannina, Ioannina, Greece
}

Received 30 April 2001; received in revised form 15 June 2001; accepted 18 June 2001

\begin{abstract}
An automated technique was developed for the detection of ischemic episodes in long duration electrocardiographic (ECG) recordings that employs an artificial neural network. In order to train the network for beat classification, a cardiac beat dataset was constructed based on recordings from the European Society of Cardiology (ESC) ST-T database. The network was trained using a Bayesian regularisation method. The raw ECG signal containing the ST segment and the T wave of each beat were the inputs to the beat classification system and the output was the classification of the beat. The input to the network was produced through a principal component analysis (PCA) to achieve dimensionality reduction. The network performance in beat classification was tested on the cardiac beat database providing $90 \%$ sensitivity ( $\mathrm{Se}$ ) and $90 \%$ specificity $(\mathrm{Sp})$. The neural beat classifier is integrated in a four-stage procedure for ischemic episode detection. The whole system was evaluated on the ESC ST-T database. When aggregate gross statistics was used the Se was $90 \%$ and the positive predictive accuracy (PPA) $89 \%$. When aggregate average statistics was used the Se became $86 \%$ and the PPA 87\%. These results are better than other reported. (C) 2002 Elsevier Science B.V. All rights reserved.
\end{abstract}

Keywords: Ischemic episode detection; Cardiac beat classification; Artificial neural networks; Bayesian regularisation

\section{Introduction}

Myocardial ischemia is defined by insufficient blood supply to the heart muscle. As a result, alterations are observed in the electrocardiographic (ECG) signal like deviations in

\footnotetext{
* Corresponding author. Tel.: +30-651-98803; fax: +30-651-98889.

E-mail address: fotiadis@cs.uoi.gr (D.I. Fotiadis).
} 
the ST segment or/and changes in the T wave [10,24]. The detection and assessment of those alterations in long duration ECGs is a simple and non-invasive method for the diagnosis of ischemia [7].

Diagnosis of myocardial ischemia by ECG is based upon two tasks: ischemic beat classification; ischemic episode definition. The first is related to the classification of beats as normal or ischemic. The accuracy of beat classification influences ischemic episode definition where sequences of ischemic beats need to be identified. Various methods have been proposed for ischemia detection based on set of rules [2,5,15,20,25-27], artificial neural networks (ANNs) [4,21,26-29], fuzzy logic [31,32] or other signal analysis techniques $[3,11,13,16,30]$.

In a previous work [23] a four-stage knowledge-based method was implemented for ischemic episode detection. This method was based on a set of medical rules for beat classification. It performed quite well, but in the case of noisy ECG recordings its positive predictive accuracy (PPA) was rather low, in comparison with the PPA of other techniques, due to the lack of flexibility that characterises rule-based systems.

In this paper an improvement of the previous method is proposed that employs ANNs for beat classification. The presence of noise affects all the measurements made in the recorded ECG signal and can lead to incorrect cardiac beat classifications. In such cases, ANNs due to their noise tolerance capabilities may perform better than the rule-based expert systems, if trained properly. Bearing this in mind, better results can be obtained by the substitution of the set of rules, in the beat classification stage of the four-stage algorithm, with a trained neural network.

\section{Materials and methods}

The neural network method for ischemic episode detection was integrated in the fourstage approach as shown in Fig. 1. It was implemented on a personal computer with two Intel PIII $450 \mathrm{MHz}$ processors and $512 \mathrm{MB}$ RAM while MATLAB ${ }^{\circledR}$ was used as the development tool. The procedure starts with the preprocessing of the recorded ECG signal in order to eliminate noise distortions like baseline wandering, $\mathrm{A} / \mathrm{C}$ interference and electromyographic contamination. Noise elimination is achieved by filtering each recorded cardiac beat separately using a signal processing procedure (details are provided in $[22,23])$. Briefly, baseline wandering is removed by subtracting from the recorded signal the first-order polynomial that best fits the cardiac beat. A/C interference and electromyographic contamination are not removed from the recorded signal but are handled properly for the detection of the $J$ point (a $20 \mathrm{~ms}$ averaging filter is applied around $J$ ). The exact location of the $J$ point is detected using a procedure based on an edge-detection algorithm [6].

Having the $J$ point detected, a neural network model, which was selected after carrying out some experiments and is described in Section 2.1, is used for classifying each cardiac beat in every lead separately. Then a sliding adaptive window technique is applied, in order to identify ischemic windows [23]. More specifically, for each ECG lead we detect intervals of approximately $30 \mathrm{~s}$ in duration (in accordance with the European Society of Cardiology (ESC) recommendations) in which more than $75 \%$ of 


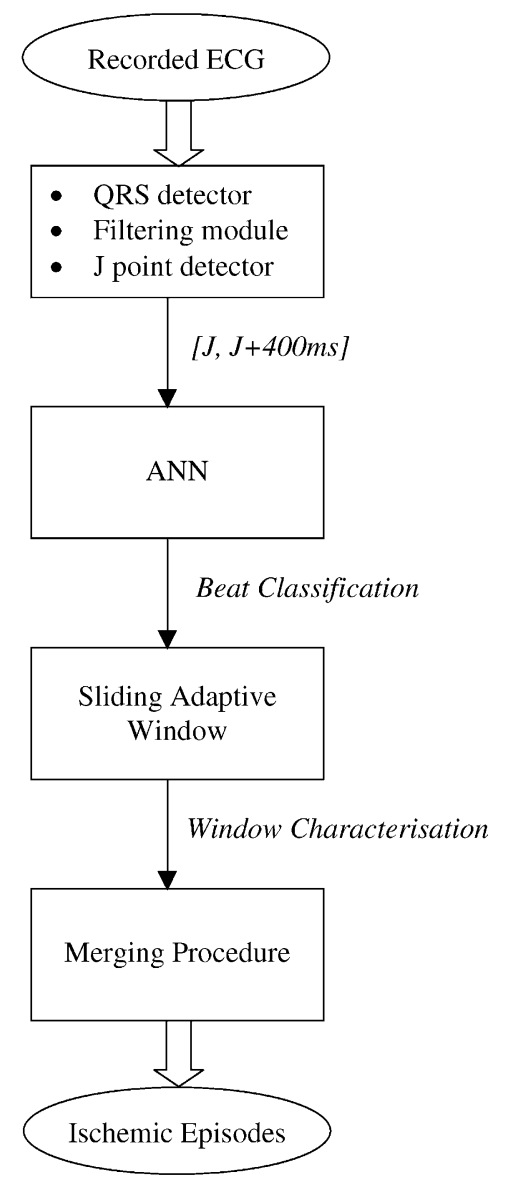

Fig. 1. The four-stage ischemic episode detection method.

the contained beats are ischemic. These intervals are characterised as ischemic windows and in the last stage all the consecutive ischemic windows are merged in order to produce the ischemic episodes in each recorded lead. In the same stage, the detected episodes in every lead are also merged and the overall ischemic episodes are defined. This means that each ECG lead is complementary to one another and the final output of the system is the margins of the ischemic episodes for each ECG recording and not for each ECG lead. The system was designed in such manner since this kind of information is more straightforward for the doctor. The $75 \%$ threshold value used for the window characterisation offers flexibility in case when the presence of noise causes misclassifications of isolated beats. For the same reason, as well as to avoid fragmentation of larger ischemic episodes, intervals of less than $20 \mathrm{~s}$ that were not characterised as ischemic windows, are permitted to intermediate between ischemic windows or episodes during the merging process. 


\subsection{Beat classification using ANN}

\subsubsection{Network architecture and training method}

For the classification of the cardiac beats a feed-forward neural network is used. The network has an input layer with four input units, one hidden layer with 10 sigmoid units (with hyperbolic tangent as activation function) and an output layer with one linear unit. The architecture of the network is shown in Fig. 2. Normally, the network could be trained to accept as input the cardiac beat and provide an output of zero for the normal and one for the ischemic case. The input pattern to the network could be the interval $[J, J+400 \mathrm{~ms}]$ (100 data points), which includes both the ST segment and the T wave. If this interval extends further than the Beat ${ }_{\text {end }}=\mathrm{QRS}+0.6 \mathrm{RR}-60 \mathrm{~ms}$ point then from this point and after the input pattern is padded with zeros. QRS denotes the location of the peak of the most prominent wave in the QRS complex and RR the time duration between two consecutive QRS points. The Beat ${ }_{\text {end }}$ point is used as a rule of thumb to define the end

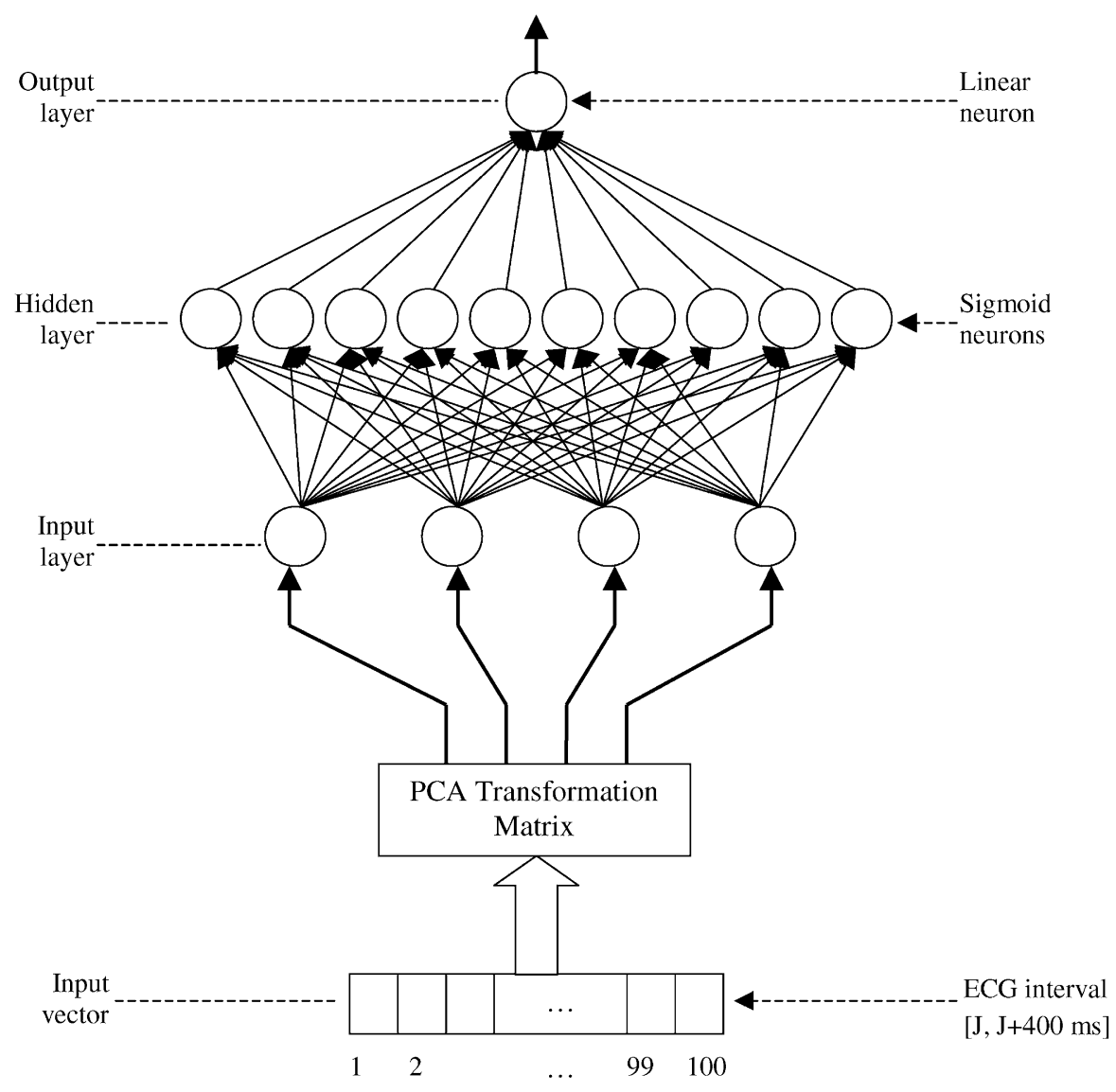

Fig. 2. The ANN architecture. 
of each presented cardiac beat. More precisely, it lies approximately $60 \mathrm{~ms}$ after the end of the $\mathrm{T}$ wave. However, in order to reduce the dimensionality of the input pattern a principal component analysis (PCA) [1] is employed to eliminate those components which contribute only a small amount (less than 5\%) to the total variance in the training set. The 5\% threshold is adopted after studying the principal components produced. The variances of the first four components, which correspond to the $95 \%$ of the total variance in the training set, have much more larger values than the rest. Thus, the input dimension is reduced to four.

Network training is performed using the Bayesian regularisation technique [9,17], which is a supervised learning method. Within this framework the following objective function is minimised:

$$
E=a_{1} \sum_{i=1}^{N}\left(t_{i}-o_{i}\right)^{2}+a_{2} \sum_{i=1}^{M} \boldsymbol{w}_{i}^{2},
$$

where $t_{i}$ are the desired network outputs, $o_{i}$ are the network outputs during training, $\boldsymbol{w}_{i}$ are the network parameters (weights and biases), $M$ is the number of those parameters and $N$ is the number of the training patterns. The hyperparameters $a_{i}$ are estimated at each iteration as follows:

$$
\begin{aligned}
& a_{1}=\frac{N-\gamma}{2 \sum_{i=1}^{N}\left(t_{i}-o_{i}\right)^{2}}, \\
& a_{2}=\frac{\gamma}{2 \sum_{i=1}^{M} \boldsymbol{w}_{i}^{2}},
\end{aligned}
$$

where $\gamma$ (called the number of effective parameters) is given as:

$$
\gamma=N-2 a_{2} \operatorname{tr}(\boldsymbol{H})^{-1}
$$

with $\boldsymbol{H}$ being the Hessian matrix of the objective function which can be approximated using the Jacobian matrix.

The network parameters $\boldsymbol{w}_{i}$ are updated according to Levenberg-Marquardt optimisation schema:

$$
\boldsymbol{w}_{i+1}=\boldsymbol{w}_{i}-\left[\boldsymbol{J}^{T} \boldsymbol{J}+\mu \boldsymbol{I}\right]^{-1} \boldsymbol{J}^{T} \boldsymbol{e},
$$

where $\boldsymbol{J}$ is the Jacobian matrix, $\boldsymbol{I}$ is the unit matrix, $\boldsymbol{e}$ is the vector of network errors and $\mu$ is a scalar parameter [12]. The parameters of the network are initialised according to the Nguyen-Widrow method [19] and $a_{1}$ and $a_{2}$ are initially set to one and zero, respectively. It should be noted that the objective function $E$ is adapted at each iteration since the hyperparameters $a_{i}$ are re-estimated.

\subsubsection{Training set construction}

In order to construct the dataset for neural network training $11 \mathrm{~h}$ of two-channel ECG recordings from the ESC ST-T database [8] were used. Three medical experts annotated independently each beat as normal, ischemic or artefact. In case of discrepancy, agreement was reached by consensus. More specifically, the experts examined the whole e0104 recording and the first hour of the e0103, e0105, e0108, e0113, e0114, e0147, e0159, $\mathrm{e} 0162$, and $\mathrm{e} 0206$ recordings (e0104 recording was used in whole since it contains a variety 
of ischemic beat patterns). This resulted in a dataset of 86,384 cardiac beats diagnosed as normal, ischemic or artefact. After removing the artefacts (6,754 beats) and the beats that were not detected by the QRS detector (2,641 beats) the final dataset is obtained with 76,989 characterised beats. From those, 1,936 beats ( 982 normal beats and 954 ischemic) are used for network training while the rest (38,344 normal beats and 36,709 ischemic) for testing the performance of the network. It must be noted that the training set corresponds to $2.5 \%$ of the final dataset and is constructed by selecting the first out of a sequence of 40 beats.

\subsection{Performance assessment of the ANN in the beat classification stage}

In order to apply the proposed beat classification method the number of units in the hidden layer of the neural network must be determined. Several experiments with different numbers of hidden units were performed. Also, other training algorithms, besides Bayesian regularisation, were studied. The network performance was evaluated using the cardiac beat test set in terms of sensitivity ( $\mathrm{Se}$ ) and specificity $(\mathrm{Sp})$. From the tests made, the network with the best performance was selected and used as the beat classifier in the second stage of the four-stage algorithm. In addition, using the cardiac beat test set the adopted ANN was also evaluated by receiver operating characteristic (ROC) analysis.

\subsection{Performance assessment of the overall method in detecting ischemic episodes}

The ANN method for beat classification was integrated into the four-stage technique for ischemic episode detection [23], replacing the rule-based classification stage. To assess the performance of the improved method Se and PPA measures were adopted. Se and PPA were calculated for both aggregate gross and average statistics. Aggregate gross statistics weights each event (episode) equally by pooling all the events over all records together, and models how the system behaves on a large number of events. Aggregate average

Table 1

Test set Se and Sp of the neural network model in classifying cardiac beats for various training algorithms and number of units in the hidden layer

\begin{tabular}{lccc}
\hline Training algorithm & No. of hidden units & $\mathrm{Se}^{\mathrm{a}}(\%)$ & $\mathrm{Sp}^{\mathrm{b}}(\%)$ \\
\hline Bayesian regularisation & 10 & 90 & 90 \\
Bayesian regularisation & 25 & 91 & 90 \\
Bayesian regularisation & 50 & 91 & 90 \\
Bayesian regularisation & 100 & 91 & 90 \\
Levenberg-Marquardt & 10 & 90 & 89 \\
Levenberg-Marquardt and use of validation set for early stopping & 10 & 89 & 88 \\
Levenberg-Marquardt & 50 & 91 & 90 \\
BFGS & 10 & 90 & 89 \\
BFGS & 25 & 90 & 89 \\
Scaled conjugate gradient & 10 & 88 & 88 \\
Scaled conjugate gradient & 25 & 90 & 90 \\
Resilient backpropagation & 10 & 89 & 88 \\
\hline
\end{tabular}

\footnotetext{
a Sensitivity.

${ }^{\mathrm{b}}$ Specificity.
} 


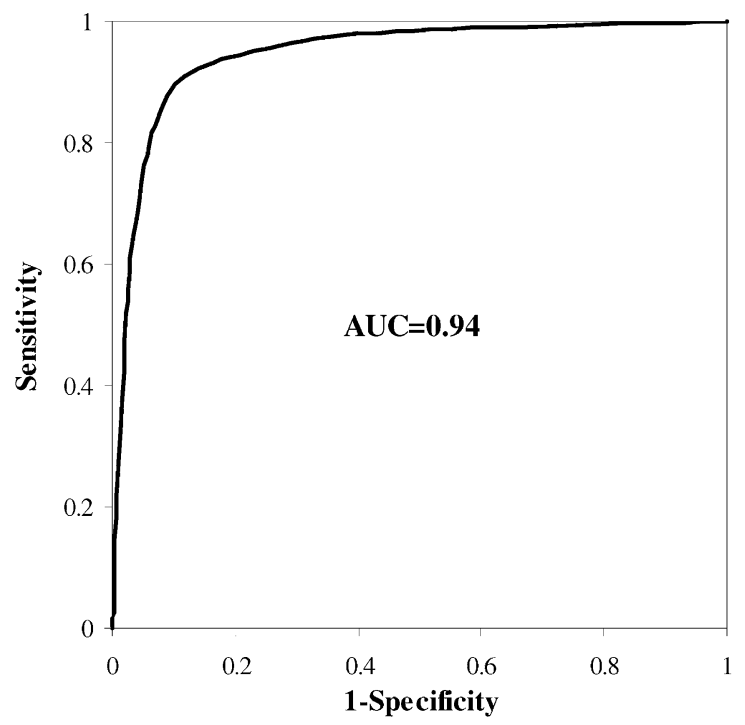

Fig. 3. ROC curve of the ANN used in the beat classification stage (Bayesian regularisation training algorithm and 10 nodes in the hidden layer). The area under the curve (AUC) is equal to 0.94 .

statistics weights each record equally, and models how the system behaves on randomly chosen records [14].

The method was tested using long duration ECG recordings, from the ESC ST-T database. This database consists of 90 continuous two-channel records, each $2 \mathrm{~h}$ duration, taken from ambulatory ECG recordings. The data in the ESC ST-T database were preprocessed in order to merge the annotated episodes of each lead. Furthermore, the annotated episodes that are referring to myocardial infarction $(\geq 0.2 \mathrm{mV}$ amplitude increase of an already positive T wave, according to $[10,24]$ ) were excluded from the merging procedure and were not taken into account during the evaluation process of the method. Also, in case of disagreement between the episodes detected from the proposed method and those annotated in the database, three medical experts examined the recordings and their diagnosis was taken into account. It is noted that other researchers have also addressed the need of refining the annotations in the ESC-ST database [30,31].

Table 2

Performance evaluation of the overall episode detection method with the ESC ST-T database using aggregate gross and average statistics ${ }^{\mathrm{a}}$

\begin{tabular}{llcc}
\hline Statistics & Value & $\mathrm{Se}^{\mathrm{b}}$ & $\mathrm{PPA}^{\mathrm{c}}$ \\
\hline Aggregate gross & Episodes & $420 / 469$ & $420 / 474$ \\
& $\%$ & 90 & 89 \\
Aggregate average & $\%$ & 86 & 87 \\
\hline
\end{tabular}

\footnotetext{
${ }^{\mathrm{a}}$ For the aggregate gross statistics, Se and PPA values are also given in terms of episodes ratios.

${ }^{\mathrm{b}}$ Sensitivity.

${ }^{\mathrm{c}}$ Positive predictive accuracy.
} 
Table 3

Performance of the episode detection method for the 90 recordings of the ESC ST-T database ${ }^{\mathrm{a}}$

\begin{tabular}{|c|c|c|c|c|c|c|c|c|c|}
\hline \multirow[t]{2}{*}{ ECG } & \multicolumn{2}{|l|}{$\mathrm{Se}^{\mathrm{b}}$} & \multicolumn{2}{|c|}{$\mathrm{PPA}^{\mathrm{c}}$} & \multirow[t]{2}{*}{ ECG } & \multicolumn{2}{|l|}{$\mathrm{Se}$} & \multicolumn{2}{|l|}{ PPA } \\
\hline & $\%$ & Episodes & $\%$ & Episodes & & $\%$ & Episodes & $\%$ & Episodes \\
\hline e0103 & 100 & $10 / 10$ & 91 & $10 / 11$ & e0211 & 100 & $1 / 1$ & 100 & $1 / 1$ \\
\hline e0104 & 88 & $7 / 8$ & 100 & $7 / 7$ & $\mathrm{e} 0212$ & 100 & $1 / 1$ & 100 & $1 / 1$ \\
\hline e0105 & 100 & $7 / 7$ & 100 & $7 / 7$ & $\mathrm{e} 0213$ & 100 & $5 / 5$ & 100 & $5 / 5$ \\
\hline e0106 & 80 & $4 / 5$ & 100 & $4 / 4$ & e0302 & 100 & $2 / 2$ & 100 & $2 / 2$ \\
\hline e0107 & 67 & $4 / 6$ & 67 & $4 / 6$ & $\mathrm{e} 0303$ & 67 & $2 / 3$ & 100 & $2 / 2$ \\
\hline e0108 & 93 & $14 / 15$ & 100 & $14 / 14$ & e0304 & 67 & $2 / 3$ & 100 & $2 / 2$ \\
\hline e0110 & 100 & $2 / 2$ & 100 & $2 / 2$ & $\mathrm{e} 0305$ & 75 & $3 / 4$ & 100 & $3 / 3$ \\
\hline e0111 & 100 & $3 / 3$ & 100 & $3 / 3$ & e0306 & 83 & $5 / 6$ & 100 & $5 / 5$ \\
\hline e0112 & 88 & $7 / 8$ & 100 & $7 / 7$ & $\mathrm{e} 0403$ & 100 & $1 / 1$ & 100 & $1 / 1$ \\
\hline $\mathrm{e} 0113$ & 89 & $8 / 9$ & 100 & $8 / 8$ & e0404 & 100 & $6 / 6$ & 100 & $6 / 6$ \\
\hline $\mathrm{e} 0114$ & 100 & $11 / 11$ & 100 & $11 / 11$ & $\mathrm{e} 0405$ & 33 & $2 / 6$ & 100 & $2 / 2$ \\
\hline e0115 & 100 & $1 / 1$ & 100 & $1 / 1$ & e0406 & 100 & $3 / 3$ & 43 & $3 / 7$ \\
\hline e0116 & 100 & $6 / 6$ & 60 & $6 / 10$ & e0408 & 0 & $0 / 1$ & 0 & $0 / 5$ \\
\hline e0118 & 88 & $7 / 8$ & 100 & $7 / 7$ & e0409 & 100 & $3 / 3$ & 100 & $3 / 3$ \\
\hline e0119 & 86 & $6 / 7$ & 60 & $6 / 10$ & $\mathrm{e} 0410$ & 100 & $7 / 7$ & 100 & $7 / 7$ \\
\hline e0121 & 91 & $10 / 11$ & 100 & $10 / 10$ & $\mathrm{e} 0411$ & 100 & $5 / 5$ & 83 & $5 / 6$ \\
\hline e0122 & 100 & $6 / 6$ & 75 & $6 / 8$ & $\mathrm{e} 0413$ & 0 & $0 / 1$ & & $0 / 0$ \\
\hline $\mathrm{e} 0123$ & 100 & $2 / 2$ & 100 & $2 / 2$ & $\mathrm{e} 0415$ & 100 & $4 / 4$ & 100 & $4 / 4$ \\
\hline $\mathrm{e} 0124$ & 20 & $1 / 5$ & 100 & $1 / 1$ & $\mathrm{e} 0417$ & 100 & $5 / 5$ & 100 & $5 / 5$ \\
\hline $\mathrm{e} 0125$ & 71 & $5 / 7$ & 100 & $5 / 5$ & $\mathrm{e} 0418$ & 89 & $8 / 9$ & 100 & $8 / 8$ \\
\hline e0126 & 67 & $2 / 3$ & 100 & $2 / 2$ & $\mathrm{e} 0501$ & 91 & $10 / 11$ & 100 & $10 / 10$ \\
\hline $\mathrm{e} 0127$ & 100 & $3 / 3$ & 50 & $3 / 6$ & e0509 & 100 & $1 / 1$ & 100 & $1 / 1$ \\
\hline e0129 & 75 & 34 & 100 & $3 / 3$ & $\mathrm{e} 0515$ & 100 & $16 / 16$ & 100 & $16 / 16$ \\
\hline e0133 & 100 & $2 / 2$ & 100 & $2 / 2$ & e0601 & 83 & $5 / 6$ & 83 & $5 / 6$ \\
\hline e0136 & 100 & $11 / 11$ & 85 & $11 / 13$ & e0602 & 50 & $1 / 2$ & 100 & $1 / 1$ \\
\hline e0139 & 100 & $1 / 1$ & 100 & $1 / 1$ & e0603 & 50 & $1 / 2$ & 100 & $1 / 1$ \\
\hline $\mathrm{e} 0147$ & 100 & $10 / 10$ & 100 & $10 / 10$ & e0604 & 100 & $7 / 7$ & 100 & $7 / 7$ \\
\hline e0148 & 88 & $7 / 8$ & 100 & $7 / 7$ & $\mathrm{e} 0605$ & 100 & $2 / 2$ & 100 & $2 / 2$ \\
\hline e0151 & 100 & $5 / 5$ & 100 & $5 / 5$ & e0606 & 67 & $2 / 3$ & 100 & $2 / 2$ \\
\hline $\mathrm{e} 0154$ & 100 & $1 / 1$ & 100 & $1 / 1$ & $\mathrm{e} 0607$ & 100 & $1 / 1$ & 100 & $1 / 1$ \\
\hline $\mathrm{e} 0155$ & 80 & $4 / 5$ & 100 & $4 / 4$ & e0609 & 89 & $8 / 9$ & 100 & $8 / 8$ \\
\hline e0159 & 0 & $0 / 1$ & & $0 / 0$ & e0610 & 100 & $6 / 6$ & 67 & $6 / 9$ \\
\hline e0161 & 100 & $2 / 2$ & 100 & $2 / 2$ & $\mathrm{e} 0611$ & 100 & $3 / 3$ & 75 & $3 / 4$ \\
\hline e0162 & 100 & $3 / 3$ & 100 & $3 / 3$ & e0612 & 100 & $6 / 6$ & 100 & $6 / 6$ \\
\hline e0163 & 100 & $5 / 5$ & 100 & $5 / 5$ & e0613 & 75 & 34 & 75 & $3 / 4$ \\
\hline e0166 & 100 & $3 / 3$ & 50 & $3 / 6$ & $\mathrm{e} 0614$ & 100 & $1 / 1$ & 100 & $1 / 1$ \\
\hline $\mathrm{e} 0170$ & 100 & $4 / 4$ & 80 & $4 / 5$ & $\mathrm{e} 0615$ & 100 & $7 / 7$ & 100 & $7 / 7$ \\
\hline e0202 & 100 & $8 / 8$ & 67 & $8 / 12$ & $\mathrm{e} 0704$ & 0 & $0 / 3$ & & $0 / 0$ \\
\hline e0203 & 100 & $7 / 7$ & 100 & $7 / 7$ & e0801 & 100 & $9 / 9$ & 82 & $9 / 11$ \\
\hline e0204 & 100 & $8 / 8$ & 80 & $8 / 10$ & e0808 & 0 & $0 / 5$ & & $0 / 0$ \\
\hline e0205 & 71 & $5 / 7$ & 100 & $5 / 5$ & e0817 & 100 & $3 / 3$ & 100 & $3 / 3$ \\
\hline e0206 & 100 & $10 / 10$ & 100 & $10 / 10$ & e0818 & 100 & $7 / 7$ & 100 & $7 / 7$ \\
\hline e0207 & 100 & $6 / 6$ & 100 & $6 / 6$ & e1301 & 100 & $5 / 5$ & 71 & $5 / 7$ \\
\hline e0208 & 100 & $10 / 10$ & 91 & $10 / 11$ & e1302 & 100 & $7 / 7$ & 100 & $7 / 7$ \\
\hline $\mathrm{e} 0210$ & 100 & $1 / 1$ & 17 & $1 / 6$ & e1304 & 100 & $2 / 2$ & 100 & $2 / 2$ \\
\hline
\end{tabular}

${ }^{\mathrm{a}}$ For each ECG recording (column, ECG) Se and PPA values are given in terms of percentages (column, \%) and episodes ratios (column, episodes).

b Sensitivity.

${ }^{\mathrm{c}}$ Positive predictive accuracy. 


\section{Results}

Table 1 displays the experimental results obtained from the use of various training algorithms and different numbers of units in the hidden layer of the neural network. The Bayesian regularisation method, described in the previous section, was found to be the most effective. All the tested neural network models performed comparably but Bayesian regularisation produced consistently slightly better results. In addition, when the number of nodes in the hidden layer was increased, the network training was very slow without significant improvement in performance. Therefore, the use of 10 hidden units was considered sufficient. For comparison, when the rule-based approach [23] was used for beat classification, the Se and the Sp were 70 and 63\%, respectively, while for the ANN method the results were 90 and $90 \%$ for both measures. The ROC analysis that was calculated for the adopted ANN (Fig. 3) produced an area under the curve equal to 0.94.

The improved episode detection method was tested on all the 90 records of the ESC ST-T database and demonstrated a Se of $90 \%$ and a PPA of $89 \%$ when aggregate gross statistics was used and 86 and $87 \%$, respectively, when aggregate average statistics was used. In Table 2 the overall performance of the method is shown while its performance per ECG recording is given in Table 3.

\section{Discussion}

Several methods for automated detection of ischemic episodes have been reported in the literature. Signal analysis techniques $[3,11,13,16,30]$ transform the original signal in order to define features appropriate for differentiation. Rule-based approaches [2,5,15,20,25-27] exhibit the highly desirable feature of interpreting their decisions. Fuzzy expert systems [31,32] manage to keep this feature without applying strict threshold values. ANNs [4,21,26-29] due to their non-linear characteristics and learning capabilities have provided good performance results. The above methods when tested with the ESC ST-T database demonstrated a Se that ranged from 71 to $94 \%$ and a PPA from 66 to $90 \%$. Some works [3$5,15,20,21,25,32]$ report better results, which are not comparable since they refer to their own datasets. In [28] a non-linear PCA neural network is proposed for ischemic beat classification instead of episode detection. Using 34 out of the 90 recordings of the ESC ST-T database, a Se of $79 \%$ and a Sp of $75 \%$ are reported.

The proposed method was evaluated using all the 90 recordings of the ESC ST-T database and exhibited very high Se and PPA (Table 4). It must be noted that the majority of the results reported in Table 4 refer to subsets of ECG recordings of the ESC ST-T database [27,29-31] and only some have used all the ESC ST-T database recordings [13,23,26]. Also, the currently evaluated beat classification network performs better than similar approaches [28], as indicated in Table 1.

The high performance of the proposed beat classification scheme can be attributed to several factors. A new cardiac beat database, based on a subset of the ESC ST-T database, was developed in collaboration with three medical experts. This database was used to train a feed-forward neural network for beat classification. During the training process values extracted from both the ST segment and the T wave were used as input to the network. The 
Table 4

Comparison of the performance of several methods for ischemic episode detection in the ESC ST-T database

\begin{tabular}{lll}
\hline Method & $\mathrm{Se}^{\mathrm{a}}(\%)$ & $\mathrm{PPA}^{\mathrm{b}}(\%)$ \\
\hline Jager et al. [13] & 85 & 86 \\
Papaloukas et al. [23] & 94 & 79 \\
Silipo and Marchesi [26] & 77 & 82 \\
Silipo and Marchesi [26] & 77 & 83 \\
Silipo and Marchesi [26] & 77 & 85 \\
Silipo and Marchesi [26] & 71 & 66 \\
Silipo and Marchesi [26] & 77 & 86 \\
Silipo et al. [27] & 85 & 88 \\
Silipo et al. [27] & 78 & 90 \\
Stamkopoulos et al. [29] & 84 & 79 \\
Taddei et al. [30] & 84 & 85 \\
Vila et al. [31] & 83 & 75 \\
Present Work & 90 & 89 \\
\hline
\end{tabular}

\footnotetext{
${ }^{a}$ Sensitivity.

${ }^{\mathrm{b}}$ Positive predictive accuracy.
}

exclusion of artefacts from the training process helped in a proper adjustment of the network's weights. In addition, a state-of-the-art neural network training algorithm based on Bayesian regularisation was employed, providing very good generalisation performance. Finally, the dimensionality reduction obtained using PCA contributed to a more effective training since the number of the network's input units was essentially decreased.

Several improvements are possible. The cardiac beat database can be expanded and include beat patterns that are not represented in the current version. Moreover, the quality of the dataset can be enhanced through the improvement of the $J$ point detection method. Inaccurate $J$ point detection results in a shift of the network's input interval that partly contains either the ST segment (the $J$ point is detected after its true location) or the T wave (the $J$ point is detected before its true location). The early detection of the $J$ point is not so decisive since we mainly need the peak of the T wave. If the $J$ point is detected after its true position then the ST segment is not fully included in the network's input vector. This obviously affects the performance of the episode detection method. For more accurate detection of the $J$ point, more advanced noise removal techniques may be examined.

\section{Conclusions and future work}

We have presented a novel method that employs ANNs for the automated detection of ischemic episodes in long duration ECG recordings. In this system a feed-forward neural network ischemic beat classifier has been implemented. In order to train the network a cardiac beat database was constructed containing beats that are annotated as ischemic, normal or artefact. The neural beat classifier was integrated into a four-stage procedure for the detection of ischemic episodes. The performance of the system was better than other reported when tested with the ESC ST-T database. 
A disadvantage of the proposed method is that it cannot provide any interpretation, for the decisions made due to the employment of the neural network model. Proper combination [18] of the knowledge-based approach [23] with the neural network model can eliminate this drawback. The potential of our method will be further assessed in recordings from ambulatory patients and patients undergoing continuous ECG monitoring in the coronary care unit.

\section{Acknowledgements}

The present work is supported by the Greek General Secretariat of Research and Technology as part of the projects "PEPER - Integrated Interface for ECG Analysis Using Advanced Methods and Result Demonstration According to ISO 9241" and "EPET IIAdvanced Techniques on Signal Processing with Applications on Medical Technology and Telecommunications".

\section{References}

[1] Akay M. Detection and estimation methods for biomedical signals. San Diego, CA: Academic Press, 1996.

[2] Akselrod S, Norymberg M, Peled I, Karabelnik E, Green MS. Computerised analysis of ST segment changes in ambulatory electrocardiograms. Med Biol Eng Comput 1987;25:513-9.

[3] Badilini F, Merri M, Benhorin J, Moss AJ. Beat-to-beat quantification and analysis of ST displacement from Holter ECGs: a new approach to ischemia detection. In: Proceedings of the IEEE Computers in Cardiology, 1992. p. 179-82.

[4] Baxt WG. Use of an artificial neural network for the diagnosis of myocardial infarction. Ann Int Med 1991;115:843-8.

[5] Cairns CB, Niemann JT, Selker HP, Laks MM. Computerised version of the time-insensitive predictive instrument: use of the Q wave, ST-segment, T wave, and patient history in the diagnosis of acute myocardial infarction by the computerised ECG. J Electrocardiol 1991;24:46-9.

[6] Daskalov IK, Dotsinsky IA, Christov II. Developments in ECG acquisition, preprocessing, parameter measurement, and recording. IEEE Eng Med Biol 1998;17:50-8.

[7] Emdin M, Taddei A, Varanini M, Raciti M, Pola S, Marchesi C, et al. Electrocardiographic and signal monitoring in ischemic heart disease: state of the art and perspective. J Med Eng Technol 1997;21:162-5.

[8] European society of cardiology, European ST-T database directory, S.T.A.R., Pisa, 1991.

[9] Foresee FD, Hagan MT. Gauss-Newton approximation to Bayesian learning. In: Proceedings of the 1997 International Joint Conference on Neural Networks, 1997;1930-35.

[10] Goldman MJ. Principles of clinical electrocardiography. 11th ed. Los Altos, CA: LANGE Medical Publications, 1982.

[11] Gramatikov B, Brinker J, Yi-chun S, Thakor NV. Wavelet analysis and time-frequency distributions of the body surface ECG before and after angioplasty. Comput Meth Program Biomed 2000;62:87-98.

[12] Hagan MT, Menhaj M. Training feedforward networks with the Marquardt algorithm. IEEE Trans Neural Networks 1994;5:989-93.

[13] Jager F, Mark RG, Moody GB, Divjak S. Analysis of transient ST segment changes during ambulatory monitoring using the Karhunen-Loève transform. In: Proceedings of the IEEE Computers in Cardiology, 1992;691-4.

[14] Jager F. Guidelines for assessing performance of ST analysers. J Med Eng Technol 1998;22:25-30.

[15] Laks MM, Cairns CB, Selker HP. An on-line computerised ECG program for the prediction of acute ischemic heart disease. In: Proceedings of the IEEE Computers in Cardiology, 1989;505-8. 
[16] Lemire D, Pharand C, Rajaonah J-C, Dube B, LeBlanc AR. Wavelet time entropy, T wave morphology and myocardial ischemia. IEEE Trans Biomed Eng 2000;47:967-70.

[17] MacKay DJC. Bayesian interpolation. Neural Comp 1992;4:415-47.

[18] Nauck D, Klawonn F, Kruse R. Foundations of neuro-fuzzy systems. Chichester: Wiley, 1997.

[19] Nguyen D, Widrow B. Improving the learning speed of 2-layer neural networks by choosing initial values of the adaptive weights. In: Proceedings of the International Joint Conference on Neural Networks, 1990;3:21-6.

[20] Oates J, Cellar B, Bernstein L, Bailey BP, Freedman SB. Real-time detection of ischemic ECG changes using quasi-orthogonal leads and artificial intelligence. In: Proceedings of the IEEE Computers in Cardiology, 1989;89-92.

[21] Ouyang N, Ikeda M, Yamauchi K. Use of an artificial neural network to analyse an ECG with QS complex in V1-V2 leads. Med Biol Eng Comput 1997;35:556-60.

[22] Papaloukas C, Fotiadis DI, Likas A, Liavas AP, Michalis LK. A robust knowledge-based technique for ischemia detection in noisy ECGs. In: Proceedings of the Fourth International Conference on KnowledgeBased Intelligent Engineering Systems and Allied Technologies, 2000;768-71.

[23] Papaloukas C, Fotiadis DI, Liavas AP, Likas A, Michalis LK. A knowledge-based technique for automated detection of ischemic episodes in long duration electrocardiograms. Med Biol Eng Comput 2001;39:10512 .

[24] Rowlands DJ. Understanding the Electrocardiogram (Section 2: Morphological Abnormalities). England: Imperial Chemical Industries PLC, 1982.

[25] Shook TL, Valvo V, Hubelbank M, Feldman CL, Stone PH, Ripley KL. Validation of a new algorithm for detection and quantification of ischemic ST segment changes during ambulatory electrocardiography. In: Proceedings of the IEEE Computers in Cardiology, 1989;57-62.

[26] Silipo R, Marchesi C. Artificial neural networks for automatic ECG analysis. IEEE Trans Signal Process 1998;46:1417-25.

[27] Silipo R, Taddei A, Marchesi C. Continuous monitoring and detection of ST-T changes in ischemic patients. In: Proceedings of the IEEE Computers in Cardiology, 1994;225-8.

[28] Stamkopoulos T, Diamantaras K, Maglaveras N, Strintzis M. ECG analysis using nonlinear PCA neural networks for ischemia detection. IEEE Trans Signal Process 1998;46:3058-67.

[29] Stamkopoulos T, Strintzis M, Pappas C, Maglaveras N. One-lead ischemia detection using a new backpropagation algorithm and the European ST-T database. In: Proceedings of the IEEE Computers in Cardiology, 1992;663-6.

[30] Taddei A, Costantino G, Silipo R, Emdin M, Marchesi C. A system for the detection of ischemic episodes in ambulatory ECG. In: Proceedings of the IEEE Computers in Cardiology, 1995;705-8.

[31] Vila J, Presedo J, Delgado M, Barro S, Ruiz R, Palacios F, et al. SUTIL: Intelligent ischemia monitoring system. Int J Med Inform 1997;47:193-214.

[32] Zahan S. A fuzzy approach to computer-assisted myocardial ischemia diagnosis. Artif Intell Med 2001; 21:271-5. 